\title{
Réflexions sur les métamorphoses récentes de la recherche biologique et médicale
}

\section{Jean Hamburger Jean-François Bach}

\section{ADRESSES}

J. Hamburger : membre de l'Académie des sciences et de l'Académie nationale de médecine. 38, rue Mazarine, 75006 Paris, France.

J.-F. Bach : membre de l'Académie des sciences. Hôpital Necker, 161, rue de Sèvres, 75015 Paris, France.

\section{TIRÉS A PART}

J. Hamburger.
1 suffit de parcourir médecine/sciences, depuis sa création en 1985, pour être convaincu que la médecine et la biologie changent peu à peu leur image.

Le changement le plus éclatant est, bien entendu, la dictature solidement établie de la biologie moléculaire. Aucun problème ne lui échappe désormais : elle est maître du jeu. Au reste sa gloire s'appuie déjà sur de merveilleux achèvements : elle légitime totalement son pouvoir absolu en métamorphosant notre regard sur toutes choses.

Première métamorphose : les matériaux de notre vie ne sont plus des êtres abstraits, définis par leurs seules fonctions, ils deviennent des molécules de composition et de structure connues, dépendant de gène(s) qui peuvent être localisés, isolés et clonés, et cette connaissance va si loin qu'elle permet souvent la fabrication de la molécule au laboratoire ou dans l'industrie et son utilisation en thérapeutique. On savait que le rein fabriquait une substance propre à stimuler la production des globules rouges, mais cette érythropoiétine était moins un corps défini qu'une fonction physiologique du rein. Aujourd'hui, l'érythropoïétine est une protéine d'un poids moléculaire de 18399 , on connaît les 166 acides aminés qui la composent, on sait tout de son gène formé de cinq exons séparés par quatre introns, on peut la fabriquer industriellement pour l'usage médical [1]. Changement à vue.

Deuxième métamorphose : on aperçoit l'intensité et la variété des communications cellulaires. Chaque cellule active de l'organisme reçoit des centaines d'informations et d'instructions provenant d'autres cellules, et en fournit d'autres à son tour [2]. Les " médiateurs" responsables de ces messages sont de mieux en mieux connus et leur nombre croît considérablement chaque année. Les récepteurs qui les accueillent ont également vu leur structure dévoilée. Et, fait remarquable, le même type de médiateurs et le même type de récepteurs se retrouvent dans des domaines différents. Le thymus forme des substances qui interviennent dans la maturation des lymphocytes, mais aussi sur l'hypophyse et le système nerveux [3]. De nombreux neuropeptides, en particulier les endorphines, agissent sur les lymphocytes et participent à un véritable réseau neuroimmuno-endocrinien. Le peptide intestinal vasoactif (VIP) a, lui aussi, des récepteurs sur les lymphocytes $\mathrm{T}$, dont il module les fonctions, et c'est également un bronchodilatateur [4]. On ne compte plus les médiateurs chimiques communs à plusieurs organes : la somatostatine n'est pas seulement formée par l'hypothalamus, mais aussi par des cellules des îlots de Langerhans du pancréas [5] ; d'autres peptides, tels que la cholécystokinine, des neurokinines 
comme la substance $\mathrm{P}$, des endomorphines sont à la fois des sécrétions du système nerveux et du tractus digestif [5]. Le concept ancien d'hormone est beaucoup trop restrictif : une hormone était définie par le tissu spécifique qui la sécrète et la cible non moins spécifique qu'elle atteint; or il apparaît aujourd'hui évident que la plupart des agents médiateurs sont formés par des tissus multiples et peuvent distribuer informations et ordres d'action à des cibles très diverses. Le monde des hormones a éclaté.

De façon plus générale, la recherche médicale autrefois centrée sur la pathologie d'organes se déplace vers une pathologie d'organisme. Et celleci prend ses racines dans la génétique moléculaire, l'immunologie moléculaire, la pharmacologie moléculaire. La cardiologie ne peut plus se limiter à l'étude du tissu cardiaque, ni la gastro-entérologie à l'étude de l'appareil digestif, ni la néphrologie à l'étude du rein.

La biologie moléculaire devient ainsi le fondement de toute recherche biologique ou médicale. Ses succès sont si remarquables que les recherches physiologiques et cliniques traditionnelles semblent perdre un peu de leur vigueur et de leur attrait. La lecture de médecine/sciences le démontre clairement. Les chercheurs subiraient-ils l'influence d'une certaine mode, comme les grands couturiers? $\mathrm{Ce}$ serait d'autant plus regrettable que le passage de l'échelle moléculaire à l'échelle du phénomène physiologique ou clinique soulève parfois de sérieux problèmes. L'un de nous (Jean Hamburger) a tenté de démontrer, dans diverses publications, qu'une césure peut se glisser entre les événements moléculaires et les phénomènes macroscopiques. C'est là, au reste, une constatation très générale dans tous les domaines de la recherche scientifique, quand on veut déduire les lois macroscopiques de ce qu'on sait à l'échelle de l'infiniment petit. En médecine, tout se passe comme si le nombre de molécules impliquées dans les manifestations de la maladie était tel que, par essence, on ne puisse intégrer la totalité des phénomènes moléculaires pour prévoir l'événement macroscopique. Sans doute est-il temps de redonner $m / s n^{\circ} 3$ vol. 6 , mars 90 force et verdeur à l'étude de la physiologie et de la pathologie à l'échelle traditionnelle de l'organisme entier.

Autre métamorphose: le cerveau n'est plus le domaine interdit qu'une carapace osseuse rendait inaccessible à une exploration physiologique et biochimique affinée. Aujourd'hui, la caméra à positons dissèque sur l'homme vivant, non seulement la structure, mais aussi les activités métaboliques de chacune des parties du système nerveux central. Le répertoire des signaux chimiques, qui permettent la communication d'un neurone à l'autre, s'accroît chaque année. Le mécanisme intime de libération des neurotransmetteurs laisse apercevoir ses secrets [6]. La psychologie et la psychiatrie s'en trouvent profondément ébranlées ; des psychoses réputées dépourvues de toute lésion organique détectable sont, en réalité, l'expression d'anomalies cérébrales précises. Peut-être même, grisée par ses succès, la neurobiologie s'expose-t-elle maintenant à quelques déceptions en prétendant " compléter la démarche de "réduction " vers le physicochimique (...) jusqu'au niveau le plus élevé qui soit : celui de la raison " [7].

On pourrait citer bien d'autres métamorphoses: la nouvelle définition biologique de la personnalité individuelle, une vision transformée du système immunologique, l'explosion de connaissances sur les maladies auto-immunes, la réussite des greffes d'organes, la maîtrise de la machinerie génétique qui a conduit l'homme au cœur même des sources de la vie, l'industrie des biotechnologies qui transforme les possibilités de fabrication de nombreux vaccins et médicaments, le pouvoir entièrement renouvelé des techniques de prévention et de diagnostic prénatal. La biologie et la médecine changent profondément méthodes, logique, pouvoir.

Qui ne se réjouirait de cette explosion de connaissances et de savoirfaire ? Elle représente sans doute la promesse d'une lutte beaucoup plus efficace contre les souffrances et les maladies des hommes. Mais le nombre croissant de découvertes originales engendre, par lui-même, un souci d'un genre nouveau. Nul ne peut désormais se targuer de maîtriser totalement les données innombrables qui s'accumulent dans une presse spécialisée de plus en plus abondante. Quand on interroge la banque de données Medline sur les articles originaux parus au cours de la seule année 1988 sur la variété de lymphocytes dite thymodépendante, on obtient une liste de 5164 titres. La même source indique que, sur une variété bien particulière de glomérulonéphrites dite à dépôts d'IgA, 791 articles ont été publiés ; sur les mécanismes de cytotoxicité immunologique (tests non compris) 15874 articles entre 1978 et 1988 ; sur la maladie polykystique des reins, 2076 articles durant la même période. Le nombre de périodiques indexés dans cette banque de données (qui est loin d'être complète) est de 2 700. Qui peut lire toutes ces références? Un besoin ignoré de nos prédécesseurs se fait donc jour : celui de publications de synthèse, qui s'efforcent d'extraire de l'immense marée des publications originales ce qui est vraiment nouveau et important, ce qui dessine les grands traits de l'avancée scientifique. En langue française, médecine/sciences est assurément, et de beaucoup, la mieux réussie de ces tentatives

\section{RÉFÉRENCES}

1. Varet B, Casadevall N, Lacombe C L'érythropoïétine. médecine/sciences 1988 ; 4 : 366-72.

2. Kordon C. Un code de la communication cellulaire. médecine/sciences $1987 ; 3$ : 126-7.

3. Modigliani E, Gattegno L. Le lymphocyte est une cellule endocrine. médecine/sciences $1987 ; 3: 275-81$.

4. Grandordy B. Neuromédiateurs bronchiques et pulmonaires. médecine/sciences 1988 ; $4: 27-35$.

5. Bataille D. Les neuropeptides de l'axe tube digestif-système nerveux central. médecine/sciences $1988 ; 4$ : 272-3.

6. Korn H. La libération des neurotransmetteurs dans le système nerveux central. médecine/sciences $1988 ; 4: 476-83$.

7. Changeux JP. De la molécule au langage : la biologie du cerveau en plein essor. médecine/sciences $1988 ; 4$ : 474-5. 